\title{
VIGILANCIA TECNOLÓGICA PARA LA ADMINISTRACIÓN DE LA INVESTIGACIÓN SOBRE CORRUPCIÓN ${ }^{\star}$ \\ TECHNOLOGICAL SURVEILLANCE FOR THE IDENTIFICATION OF LINES OF RESEARCH OVER CORRUPTION
}

Recibido: 29 de agosto de 2019

Evaluado: 28 de septiembre de 2019

Aprobado: 15 de noviembre de 2019

Diana Stefany Aya Palencia**

Universidad Surcolombiana

Orcid: 0000-0002-7374-0881

Nazly Alejandra Fernández Gómez

Universidad Surcolombiana

Orcid: 0000-0002-3821-6021

Cómo citar este artículo: Aya Paencia, D. S. y Fernández Gómez, N. A. (2020). Vigilancia tecnológica para la administración de la investigación sobre corrupción. Revista Estrategia Organizacional, 9(1). doi: https://doi.org/10.22490/25392786.3643

* $\quad$ Artículo de investigación.

** Universidad Surcolombiana.

Contacto CV LAc: 0000152783201712181912 


\section{RESUMEN}

Introducción: El desarrollo científico que aborda el tema de la corrupción resulta fundamental para lograr una adecuada identificación de líneas de investigación para grupos que pretendan abordar este tema como objeto de estudio. Metodología: Con la ayuda de la base de datos Scopus, utilizando la interfaz biblioshiny del paquete bibliometrix del software libre R-Project, se pudo canalizar artículos de gran impacto que ayudo a definir líneas investigativas o referenciar palabras claves que son de gran importancia para poder definirlas, este proceso no solo puede ser utilizado en la línea de corrupción, dado que aplica para cualquier tema de interés; como salud, educación, pobreza, alimentación, entre otros, lo importante es definir un tema macro del cual se desprendan ramas que lo afectan directa o indirectamente. Por lo anterior, el grupo de investigación Statistics and Probability, utilizó como herramienta principal el motor de búsqueda de bases de datos SCOPUS bajo la línea de corrupción, que permitió realizar una cadena referencial donde indica el paso a paso para llegar a un resultado. Resultados: Finalmente se obtuvo como resultado, que el proceso para definir líneas de investigación a partir del uso de bases de datos es un instrumento que permite llegar a información de fuentes primarias lo que garantiza un resultado con referencias verídicas que pueden ser utilizadas para citar los proyectos a investigar.

Palabras clave: admnistración, investigación, corrupción, desarrollo, inversión.

\section{ABSTRACT}

The scientific development that addresses the issue of corruption is fundamental to achieve an adequate identification of lines of research of groups that intend to address this issue as a subject of study. With the help of the Scopus database, using the biblioshiny interface of the bibliometrix package of the free software R-Project, it was possible to channel articles of great impact that helped define research lines or reference key words that are of great importance in order to define them. This process can not only be used in the corruption line, given that it applies to any topic of interest; such as health, education, poverty, food, among others, the important thing is to define a macro topic from which branches that affect it directly or indirectly can be derived. For this reason, the research group Statistics and Probability, used as a main tool the database search engine SCOPUS under the corruption line, which allowed making a reference chain where it indicates the step by step to reach a result. Finally, it was obtained as a result, that the process to define lines of investigation from the use of databases is an 
instrument that allows reaching information from primary sources which guarantees a result with true references that can be used to cite the projects to be investigated.

Keywords: administration, research, corruption, development, investment, seedbed.

\section{INTRODUCCIÓN}

La constante evolución tecnológica y científica a nivel mundial transforma las maneras como se investiga, lo que hace necesario apropiar y aplicar métodos que permitan la identificación de líneas que puedan potenciar el trabajo de semilleros, en términos de mejorar su capacidad de generación de conocimiento, de cara a la vanguardia de la investigación global en el tema de estudio.

Teniendo en cuenta lo anterior, la vigilancia tecnológica se convierte en una herramienta clave para la definición de lo prioritario a la hora de investigar. En tal sentido, desde el año 2018 se vienen ejecutando una serie de actividades en torno al desarrollo de estas capacidades de observación del entorno científico, captación de información y toma de decisiones, en el Semillero de Investigación Statistics and Probability, de la Facultad de Economía y Administración de la Universidad Surcolombiana. El lograr tener líneas de investigación no es tarea fácil, pues hay un interés común que debe ser orientado a las prioridades para investigar; en este sentido, se describe el aporte científico que brinda la herramienta Scopus para la identificación de líneas de investigación.

De este modo, en este artículo se presentan los resultados de un ejercicio de revisión científica que examina la información relevante y necesaria que concierne al tema de la corrupción, propuesto para el semillero de investigación Statistics and Probability.

El artículo está dividido en seis apartados -incluida esta introducción-; después, se realiza una fundamentación teórica de los conceptos de "vigilancia tecnológica" y "líneas de investigación"; luego, se presenta la metodología para llevar a cabo el propósito del estudio; a continuación, se muestran los resultados de los análisis llevados a cabo con sus respectivas discusiones derivadas; y finalmente, se proponen unas conclusiones y se relacionan las referencias bibliográficas. 


\section{METODOLOGÍA}

“La Vigilancia Tecnológica es un proceso organizado, selectivo y permanente, de captar información del exterior y de la propia organización sobre ciencia y tecnología, seleccionarla, analizarla, difundirla y comunicarla, para convertirla en conocimiento para tomar decisiones con menor riesgo y poder anticiparse a los cambios." Norma UNE 166006:2006

En ese contexto, se realizó una extracción de la información bibliográfica de los documentos sobre la corrupción publicados en el período de 1989 a 2018 de la base bibliográfica SCOPUS; llevando a cabo, de esta manera, una investigación de tipo descriptivo, con las siguientes etapas:

\section{IDENTIFICACIÓN DE NECESIDADES}

En esta etapa se seleccionaron factores críticos de vigilancia para este estudio y se definió la ficha de vigilancia tecnológica con las palabras claves relacionadas con la temática de estudio y las fuentes de consulta.

Tabla 1. Ficha de vigilancia tecnológica.

\begin{tabular}{|c|l|l|}
\hline \multirow{2}{*}{ Palabras clave } & Español & Inglés \\
\cline { 2 - 3 } & Corrupción & Corruption \\
\hline Fuentes de Consulta & SCOPUS \\
\hline
\end{tabular}

Fuente: elaboración propia

\section{BÚSQUEDA Y CAPTACIÓN DE INFORMACIÓN}

En esta fase, se construyó y operó la ecuación de búsqueda TITLE ( corruption ) AND ( LIMIT-TO ( DOCTYPE, "ar") OR LIMIT-TO ( DOCTYPE , "re" )) AND ( LIMIT-TO ( SUBJAREA, "ECON" )) AND ( LIMIT-TO ( EXACTKEYWORD, “Corruption")) AND ( LIMIT-TO ( LANGUAGE , "English") OR LIMIT-TO ( LANGUAGE , "Spanish") ) en la base de datos estructurada SCOPUS.

A partir de los criterios referenciados en el párrafo anterior, se obtuvieron los siguientes resultados: 
Tabla 2. Resultados de la búsqueda y captación de información.

\begin{tabular}{|l|r|}
\hline \multicolumn{1}{|c|}{ Descripción } & Resultados \\
\hline Documentos & 1.057 \\
\hline Fuentes de Información (Revistas, Libros, etc.) & 295 \\
\hline Palabras claves de los autores & 1855 \\
\hline Periódo & $1989-2018$ \\
\hline Promedio de citaciones por documento & 22.16 \\
\hline Autores & 1742 \\
\hline Autores de documentos de autoría individual & 273 \\
\hline Autores de documentos de multiautoría & 1469 \\
\hline Documentos de autoría individual & 356 \\
\hline Documentos por autor & 0.607 \\
\hline Autores por documento & 1.65 \\
\hline Co-autores por document & 2.04 \\
\hline Índice de colaboración & 2.1 \\
\hline & \\
\hline Tipos de document & 1018 \\
\hline Artículos & 39 \\
\hline Revisiones & \\
\hline
\end{tabular}

Fuente: elaboración propia

\section{ANÁLISIS CIENCIOMÉTRICO}

Con base en los resultados obtenidos a partir de la operación de la ecuación de búsqueda en la base de datos estructurada SCOPUS, se realizó el análisis cienciométrico de las publicaciones, lo que permite gestionar y extraer conocimiento crítico de los procesos de búsqueda. El análisis se realizó utilizando la interfaz biblioshiny del paquete bibliometrix del software libre R-Project.

\section{RESULTADOS}

\section{DINÁMICA DE PUBLICACIONES}

En esta sección se expone la dinámica de las publicaciones relacionadas con el tema objeto de este estudio, en términos de: publicaciones por año y principales temas de interés. 


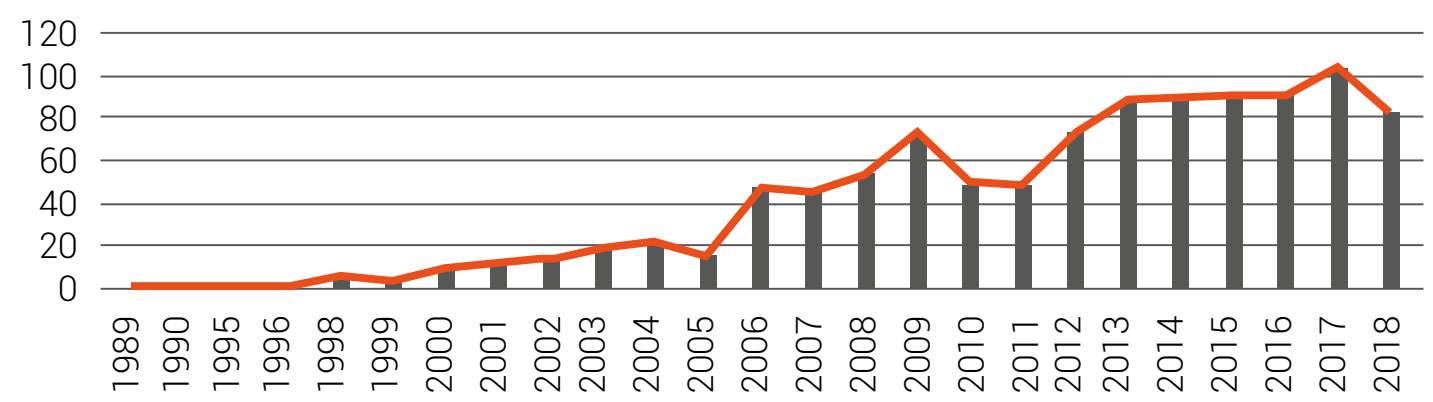

Figura 1. Número de publicaciones por año.

Fuente: elaboración propia con base en información de SCOPUS

Con respecto al número de publicaciones por año relacionadas con el tema de estudio, como puede verse, para el periodo de tiempo graficado (1989-2018), se mantuvo, en términos generales, una tendencia creciente del interés de los investigadores sobre el tema. En ese sentido, más del $80 \%$ de las publicaciones se llevaron a cabo durante los últimos once años (entre 2008 y 2018).

\section{TEMAS DE INVESTIGACIÓN POR AÑO}

Los temas (palabras clave) catalogados como destacados porque muestran presencia en mayor número de publicaciones en el tiempo son:

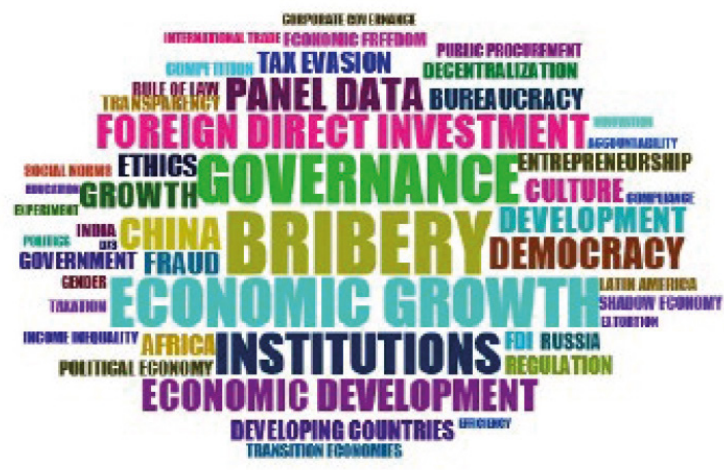

Figura 2. Nube de palabras claves

Fuente: elaboración propia con base en información de SCOPUS, a través de biblioshiny. 


\section{PRINCIPALES TEMAS DE INVESTIGACIÓN}

Los principales temas de investigación identificados pueden verse en el Figura 3 :

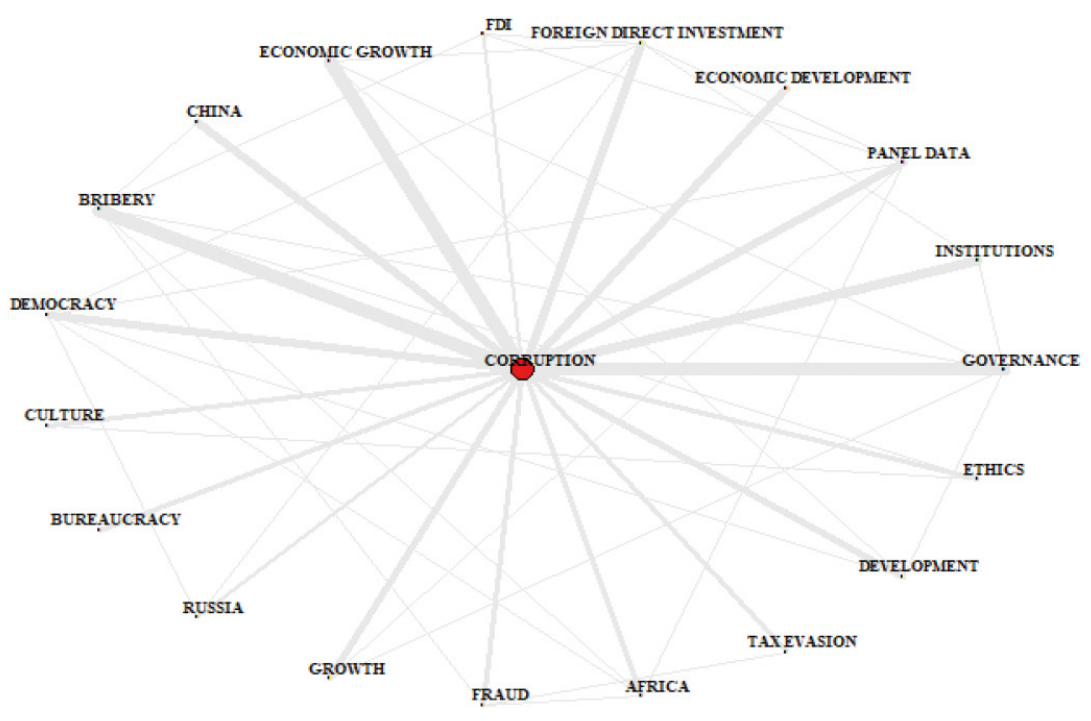

Figura 3. Mapa de correlación entre temas.

Fuente: elaboración propia con base en información de SCOPUS, a través de biblioshiny.

De esta manera, se puede ver cómo los principales términos relacionados con la palabra clave "soborno" son: China, Inversión Extranjera Directa, Gobernanza, Ética, África y Fraude; mientras que para "Crecimiento Económico" son, además de Inversión Extranjera Directa y Gobernanza, Desarrollo; de esta misma forma, con "Gobernanza" se relacionan más los términos Instituciones, Crecimiento y Soborno; "Instituciones" con Inversión Extranjera Directa y Gobernanza; "Inversión Extranjera Directa" con Crecimiento Económico, Democracia, Instituciones y Panel de Datos; "Panel de Datos" con Inversión Extranjera Directa, Democracia, Crecimiento y África; "Democracia" con Inversión Extranjera Directa, Panel de Datos, Desarrollo, África y Rusia; "Desarrollo" con Democracia y Crecimiento Económico; "Crecimiento" con Panel de Datos y Gobernanza; "Fraude" con Soborno, Evasión de Impuestos y África; y "Cultura" con Ética. 
De otro lado, se aplicó la técnica Análisis de Correspondencias a las palabras claves con el objetivo de mapear la estructura del marco conceptual, es decir, para identificar grupos de documentos que expresan conceptos comunes, utilizando las co-ocurrencias de palabras claves en la colección bibliográfica estudiada. Los resultados se trazan en un mapa bidimensional (Figura 4).

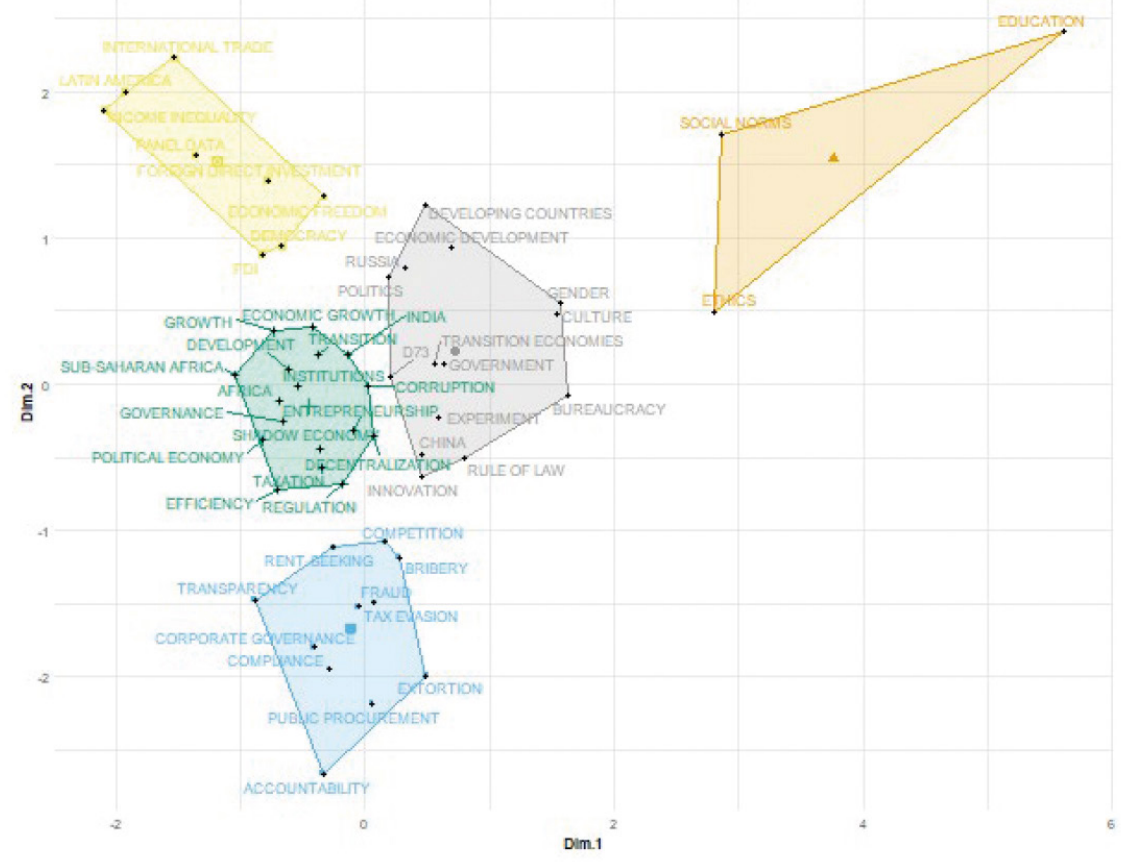

Figura 4. Mapa de estructura conceptual.

Fuente: elaboración propia con base en información de SCOPUS, a través de biblioshiny.

En ese contexto, a partir del Figura 4, se pueden identificar 5 redes de palabras claves, agrupadas por colores, las cuales son las líneas de investigación propuestas para el semillero Probability \& Statistics:

- Azul - "anticorrupción": Soborno, fraude, evasión de impuestos, extorsión, contratación pública, contabilidad, complicidad, gobierno corporativo, transparencia, búsqueda de rentas y competencia. 
- Verde - "corrupción y crecimiento económico": Crecimiento económico, transición, India, instituciones, emprendimiento, economía en la sombra, descentralización, regulación, impuestos, eficiencia, economía política, gobernanza, África, Desarrollo.

- Gris - "corrupción y desarrollo": Países en desarrollo, desarrollo económico, género, cultura, economía de transición, gobierno, burocracia, experimento, China, imperio de la ley, innovación, política, Rusia.

- Naranja: Educación, ética y normas sociales.

- Amarillo - "Corrupción, libertad económica y democracia": Comercio internacional, Latinoamérica, desigualdad en el ingreso, panel de datos, inversión extranjera directa, libertad económica y democracia.

Con respecto a lo que se ha investigado en cada línea, se presenta la siguiente tabla que relaciona artículos científicos que pueden servir de referencia para futuras publicaciones del semillero: 


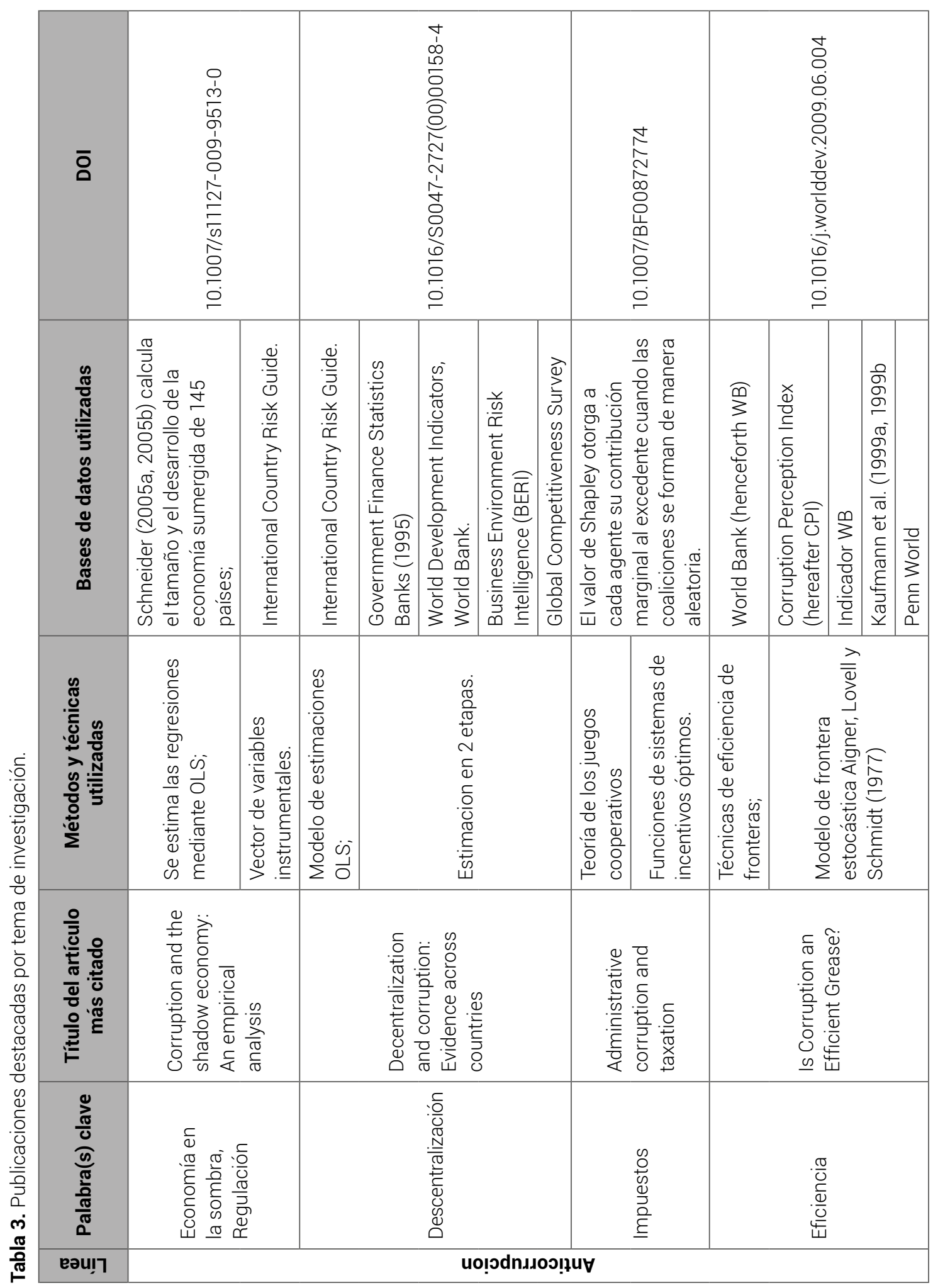




\begin{tabular}{|c|c|c|c|c|c|c|c|c|c|c|c|c|c|}
\hline$\overline{\mathrm{o}}$ & \multicolumn{2}{|c|}{\begin{tabular}{l}
$\overline{8}$ \\
0 \\
0 \\
0 \\
00 \\
0 \\
0 \\
0 \\
0 \\
0 \\
0 \\
0 \\
0 \\
0 \\
\hdashline \\
0 \\
0 \\
0 \\
0 \\
0 \\
\end{tabular}} & \multirow{2}{*}{ 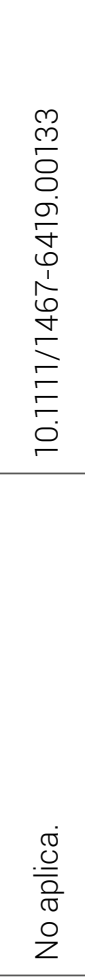 } & \multirow{2}{*}{ 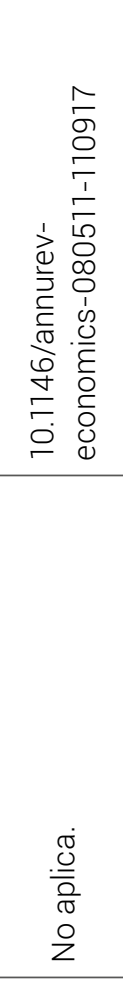 } & \multicolumn{3}{|c|}{$\begin{array}{l}1 \\
\text { Dे } \\
0 \\
0 \\
0 \\
0 \\
0 \\
0 \\
0 \\
0 \\
0 \\
1 \\
0 \\
0 \\
0 \\
0 \\
0 \\
0 \\
0 \\
0 \\
0 \\
0\end{array}$} & \multirow{2}{*}{ 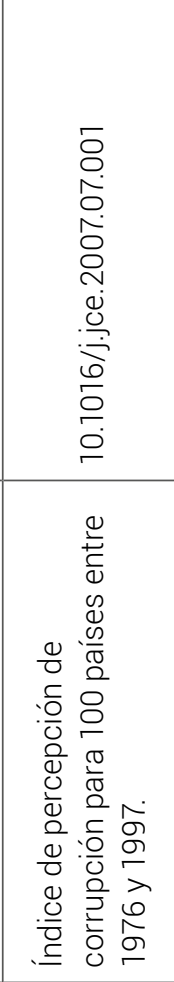 } & \multicolumn{3}{|c|}{ 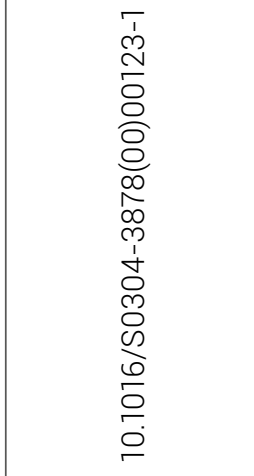 } & & 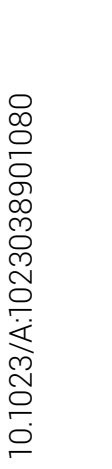 \\
\hline 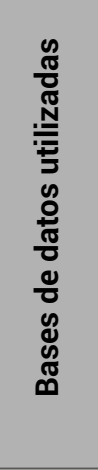 & \multicolumn{2}{|c|}{ 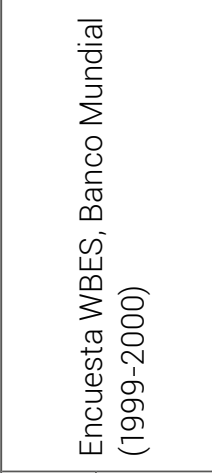 } & & & $\begin{array}{l}0 \\
0 \\
\frac{0}{0} \\
\frac{0}{0} \\
\frac{1}{0} \\
\frac{0}{0} \\
\frac{0}{0} \\
0\end{array}$ & 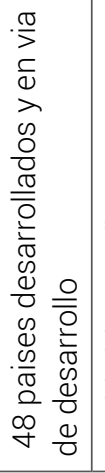 & 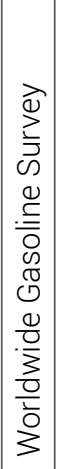 & & 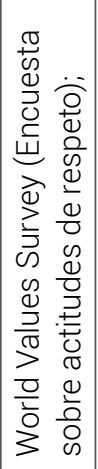 & 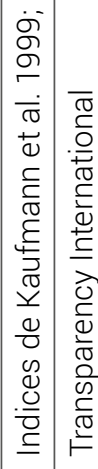 & 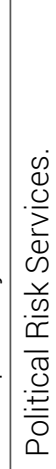 & & 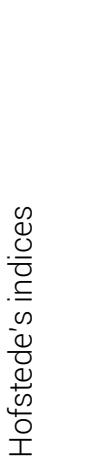 \\
\hline 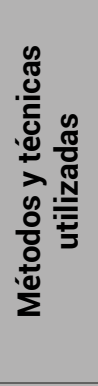 & 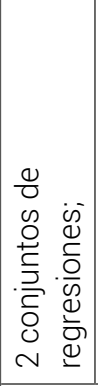 & $\begin{array}{l}\frac{\dot{0}}{0} \\
\frac{0}{0} \\
\frac{0}{0} \\
\frac{0}{0} \\
\frac{0}{2} \\
\frac{0}{2}\end{array}$ & 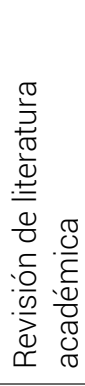 & 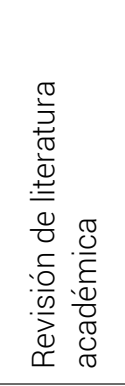 & 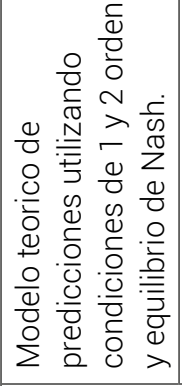 & 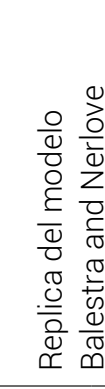 & & 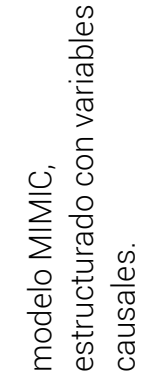 & 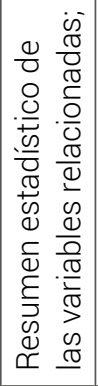 & $\begin{array}{l}\frac{1}{0} \\
\frac{0}{0} \\
\frac{\pi}{0} \\
\frac{0}{0} \\
0 \\
\frac{0}{0} \\
\frac{0}{0} \\
\frac{0}{0} \\
\frac{0}{2}\end{array}$ & & 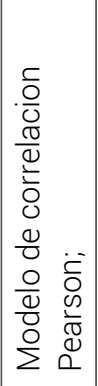 & 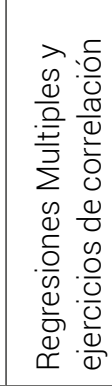 \\
\hline 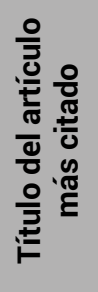 & \multicolumn{2}{|c|}{ 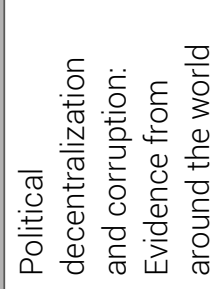 } & 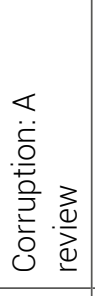 & 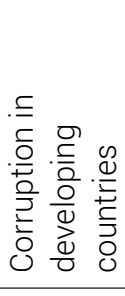 & \multicolumn{3}{|c|}{ 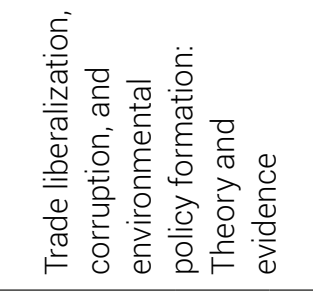 } & 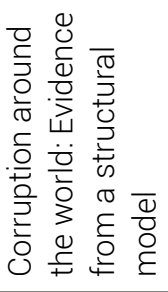 & \multicolumn{3}{|c|}{ 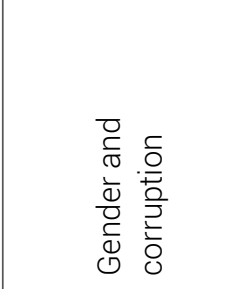 } & \multicolumn{2}{|c|}{ 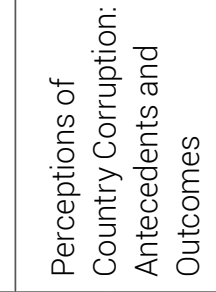 } \\
\hline $\begin{array}{l}\frac{0}{\pi} \\
\frac{\pi}{0} \\
\frac{\pi}{\pi} \\
\frac{0}{0} \\
\frac{\pi}{0} \\
0\end{array}$ & \multicolumn{2}{|c|}{ 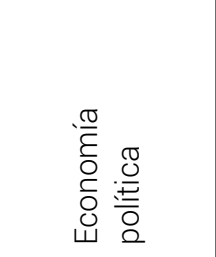 } & 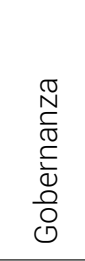 & $\begin{array}{l}\stackrel{O}{0} \\
\stackrel{\overline{0}}{\overline{0}} \\
0 \\
\mathscr{D} \\
0\end{array}$ & \multicolumn{3}{|c|}{ 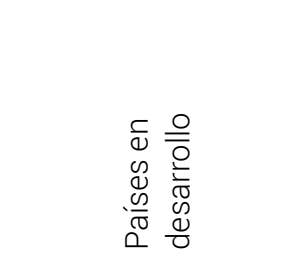 } & 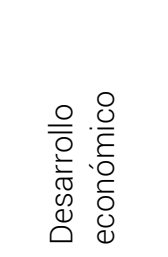 & & 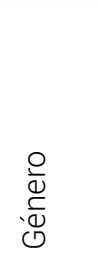 & & & $\begin{array}{l}\stackrel{\frac{0}{J}}{ \pm} \\
\frac{ \pm}{J} \\
0\end{array}$ \\
\hline eəu!า & \multicolumn{4}{|c|}{ uo!̣odnıرoग!̣u } & \multicolumn{7}{|c|}{ O||0גנesəp K uọ!odnגנoכ } & & \\
\hline
\end{tabular}




\begin{tabular}{|c|c|c|c|c|c|c|c|c|c|c|}
\hline $\bar{\circ}$ & d & 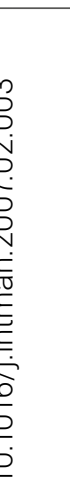 & & & \multicolumn{2}{|c|}{$\begin{array}{l}x \\
0 \\
0 \\
0 \\
0 \\
0 \\
0 \\
0 \\
o 0 \\
0 \\
N \\
1 \\
0 \\
0 \\
0 \\
0 \\
0 \\
0 \\
0 \\
\end{array}$} & \multicolumn{2}{|c|}{$\begin{array}{l}0 \\
0 \\
\infty^{2} \\
\overline{0} \\
0 \\
0 \\
0 \\
0 \\
0 \\
\infty \\
0 \\
1 \\
0 \\
0 \\
0 \\
0 \\
0 \\
0 \\
0 \\
0 \\
0 \\
0\end{array}$} & 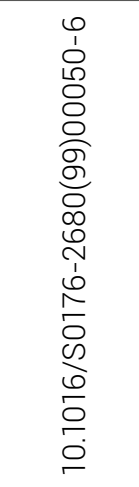 & 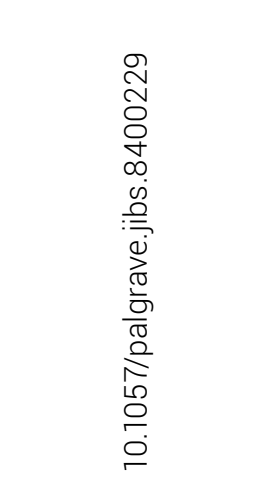 \\
\hline 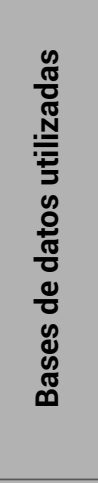 & 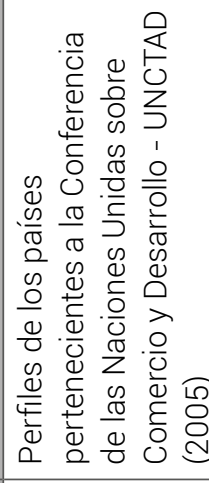 & 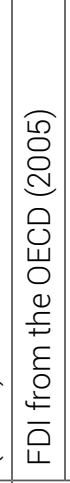 & $\mid$ & 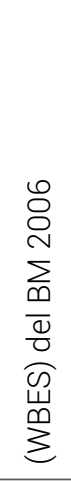 & 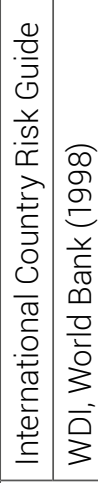 & 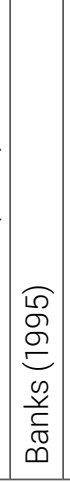 & 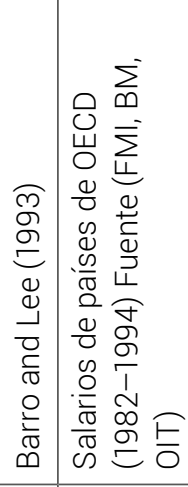 & 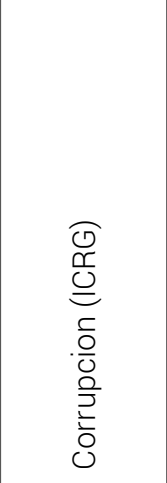 & 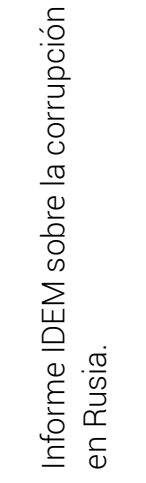 & $\begin{array}{l}\frac{\pi}{0} \\
\frac{0}{0} \\
\frac{0}{0} \\
\frac{0}{z}\end{array}$ \\
\hline 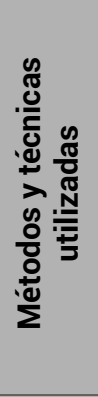 & $\begin{array}{l}\dot{\theta} \\
8 \\
0 \\
\stackrel{0}{0} \\
\dot{0} \\
\frac{0}{0} \\
\frac{0}{0} \\
\frac{1}{0} \\
\frac{\overline{0}}{0} \\
\frac{0}{5}\end{array}$ & \multicolumn{3}{|c|}{ 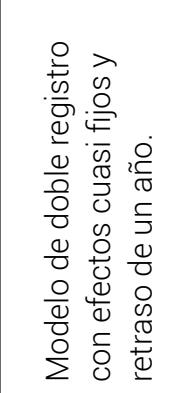 } & \multicolumn{2}{|c|}{$\begin{array}{l}\frac{9}{0} \\
\frac{0}{0} \\
\frac{0}{0} \\
\frac{0}{2}\end{array}$} & $\begin{array}{l}\dot{i} \\
\dot{0} \\
\frac{0}{Q} \\
\dot{0} \\
\dot{0}\end{array}$ & 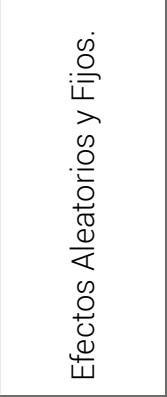 & 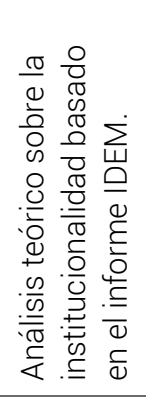 & 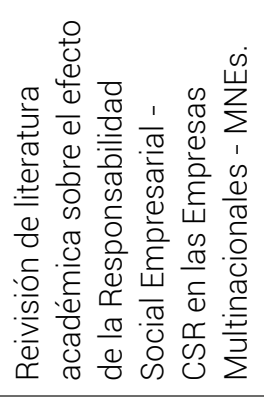 \\
\hline 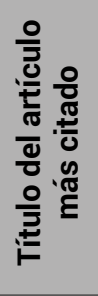 & \multicolumn{4}{|c|}{ 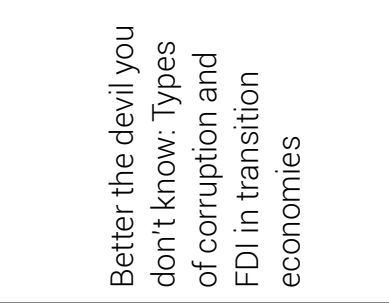 } & \multicolumn{2}{|c|}{ 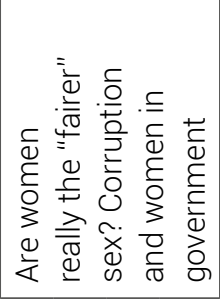 } & \multicolumn{2}{|c|}{ 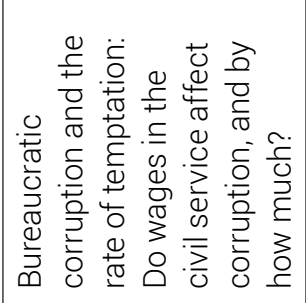 } & 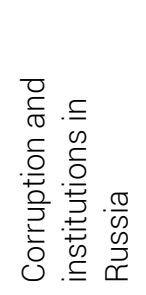 & 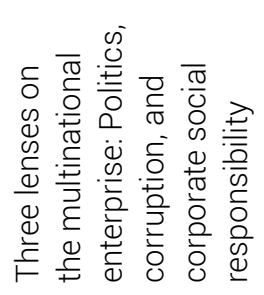 \\
\hline $\begin{array}{l}\frac{0}{\pi} \\
\frac{\pi}{0} \\
\frac{\pi}{\pi} \\
\frac{\pi}{0} \\
\frac{\pi}{0}\end{array}$ & \multicolumn{4}{|c|}{ 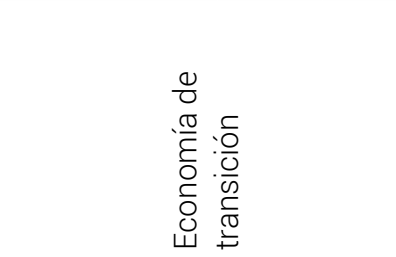 } & \multicolumn{2}{|c|}{$\begin{array}{l}\frac{0}{\frac{1}{4}} \\
\frac{0}{0} \\
\frac{0}{0} \\
0\end{array}$} & & $\begin{array}{l}\frac{\pi}{0} \\
\frac{\pi}{0} \\
\frac{0}{3} \\
0 \\
0\end{array}$ & 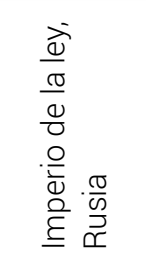 & 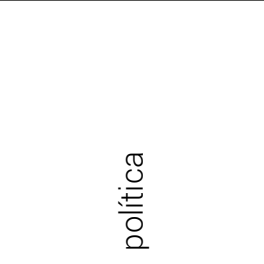 \\
\hline eəu! & \multicolumn{10}{|c|}{ 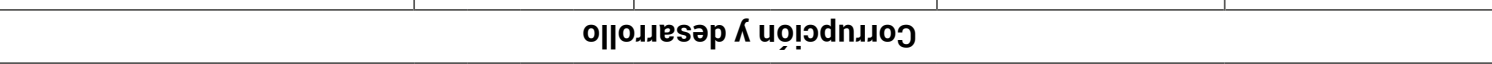 } \\
\hline
\end{tabular}




\begin{tabular}{|c|c|c|c|c|c|c|c|c|c|c|c|}
\hline סㅁㅁ & 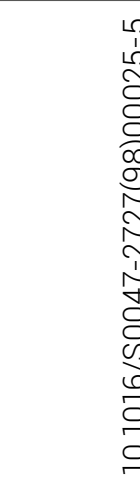 & & 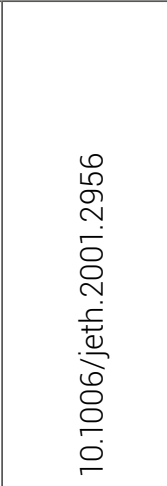 & 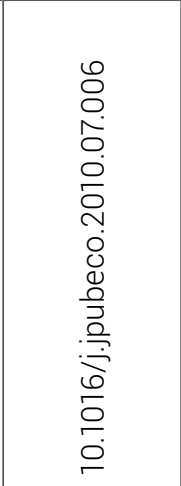 & \multicolumn{2}{|l|}{ 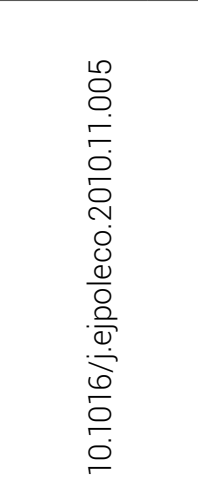 } & \multicolumn{2}{|c|}{ 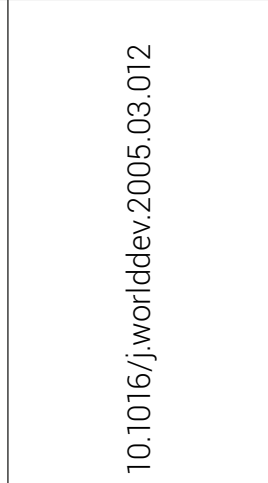 } & \multicolumn{2}{|r|}{ 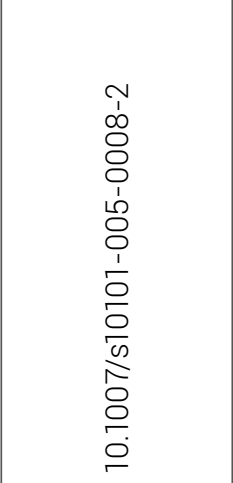 } & \multirow[b]{2}{*}{ 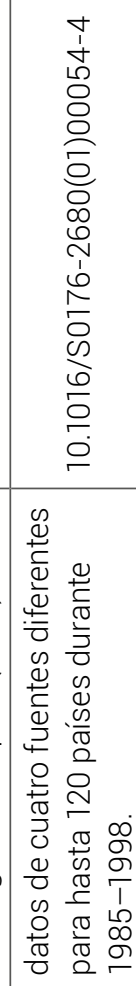 } \\
\hline 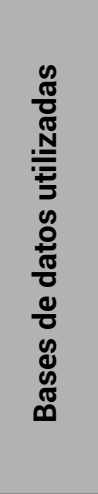 & 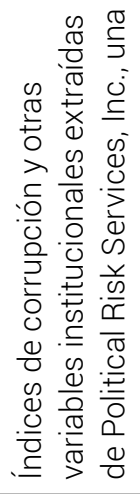 & 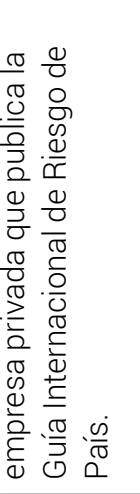 & 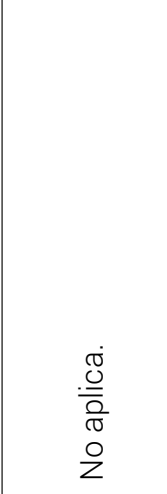 & 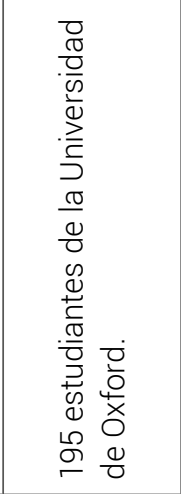 & 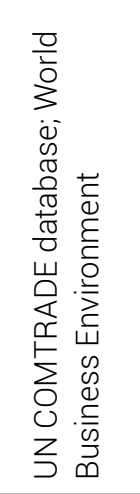 & 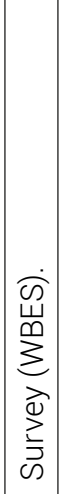 & 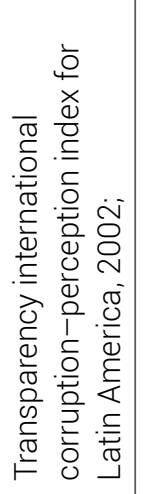 & 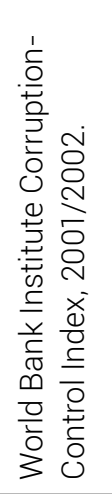 & 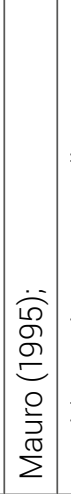 & 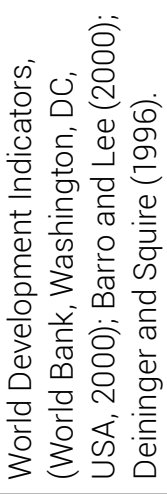 & \\
\hline 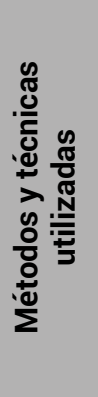 & 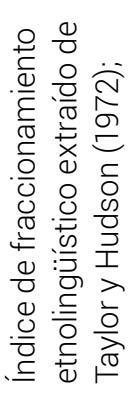 & 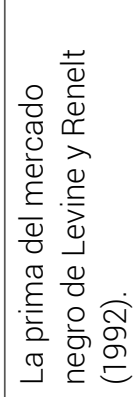 & 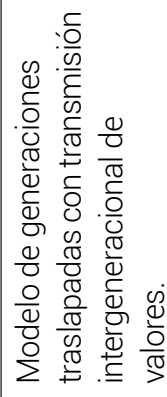 & 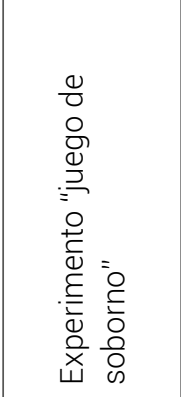 & 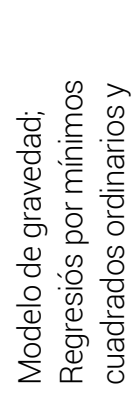 & & \multicolumn{2}{|c|}{ 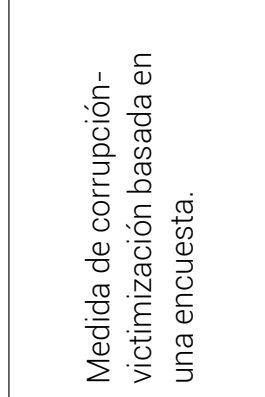 } & & 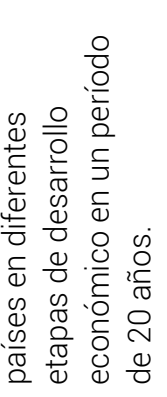 & 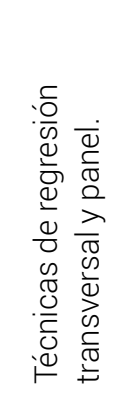 \\
\hline 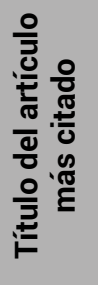 & \multicolumn{2}{|c|}{ 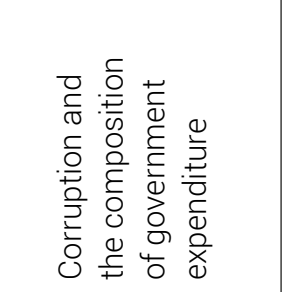 } & 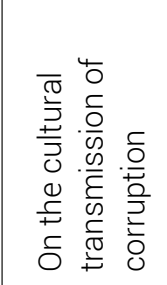 & 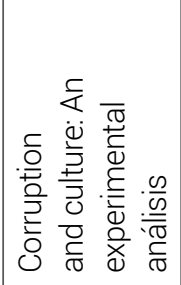 & 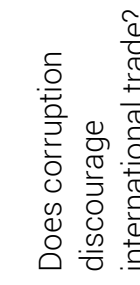 & & \multicolumn{2}{|c|}{ 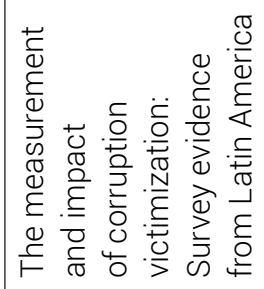 } & 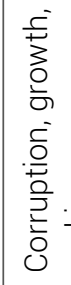 & 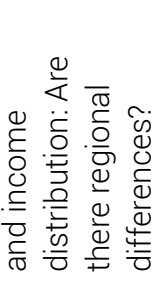 & 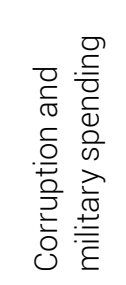 \\
\hline $\begin{array}{l}\frac{0}{\pi} \\
\frac{\pi}{0} \\
\frac{\pi}{\pi} \\
\frac{\pi}{\frac{\pi}{\pi}} \\
\frac{\pi}{0}\end{array}$ & \multicolumn{2}{|c|}{ 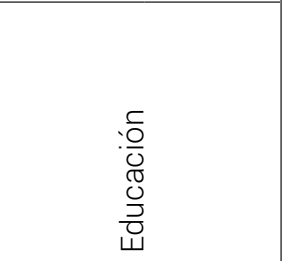 } & 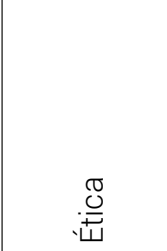 & 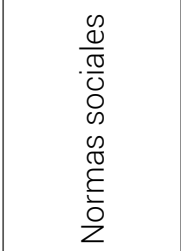 & 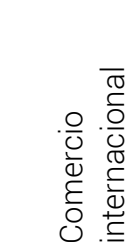 & & 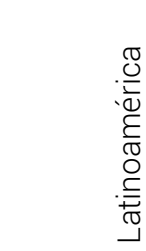 & & & 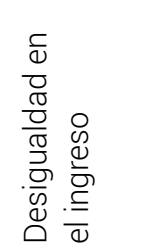 & $\begin{array}{l}0 \\
0 \\
0 \\
\frac{0}{0} \\
0 \\
0 \\
0 \\
0 \\
\frac{0}{0} \\
0 \\
0\end{array}$ \\
\hline eəu!n & \multicolumn{4}{|c|}{ 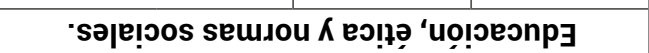 } & \multicolumn{7}{|c|}{ 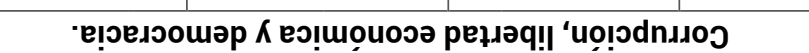 } \\
\hline
\end{tabular}




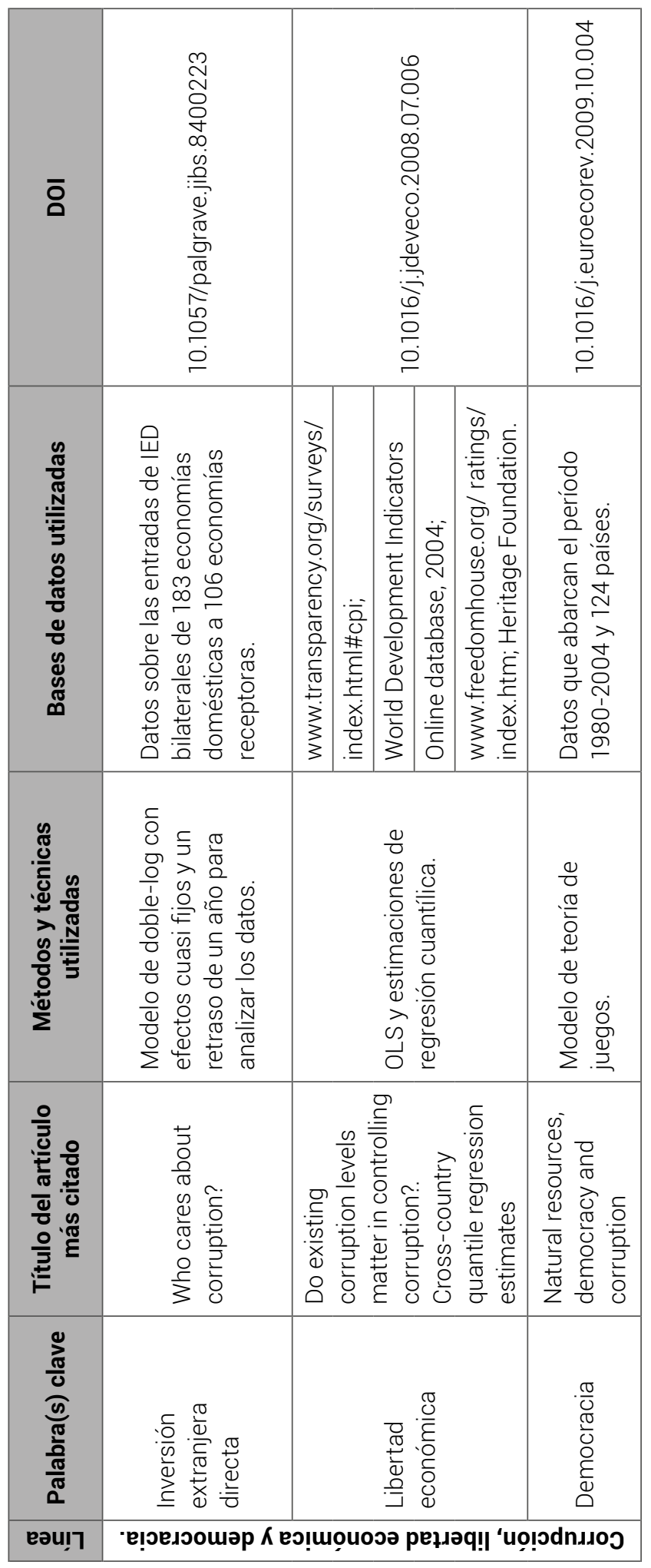

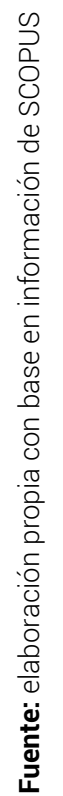


En términos generales, de acuerdo a la relación de publicaciones presentadas, se puede evidenciar una preocupación cada vez mayor de los investigadores por encontrar la explicación de las causas de la corrupción, a partir de la construcción de marcos y modelos teóricos, correlacionando, en algunos casos, la corrupción con otras variables como género. En ese contexto, las publicaciones evalúan desde la eficiencia de la autorregulación como herramienta anticorrupción, pasando por el análisis de las causas de la corrupción por medio de la gestión de datos de muchos países, hasta enfoques en los que la corrupción aporta al crecimiento económico.

Algunos temas destacados por el interés de los investigadores por ellos son: soborno, corrupción política, instituciones, desarrollo económico, ética, educación y aprendizaje transformativo. De esta manera, para el semillero de investigación, también es importante identificar cuáles son las fuentes (revistas, editoriales, bases de datos académicas, bases de datos públicas, entre otras) claves que se dedican a difundir el conocimiento generado alrededor del tema objeto de estudio.

\section{ACTORES LÍDERES}

En esta sección se presentan los resultados de un ejercicio de identificación de actores clave para los intereses del estudio.

\section{INVESTIGADORES}

Los principales investigadores identificados, pueden verse en el siguiente Figura:

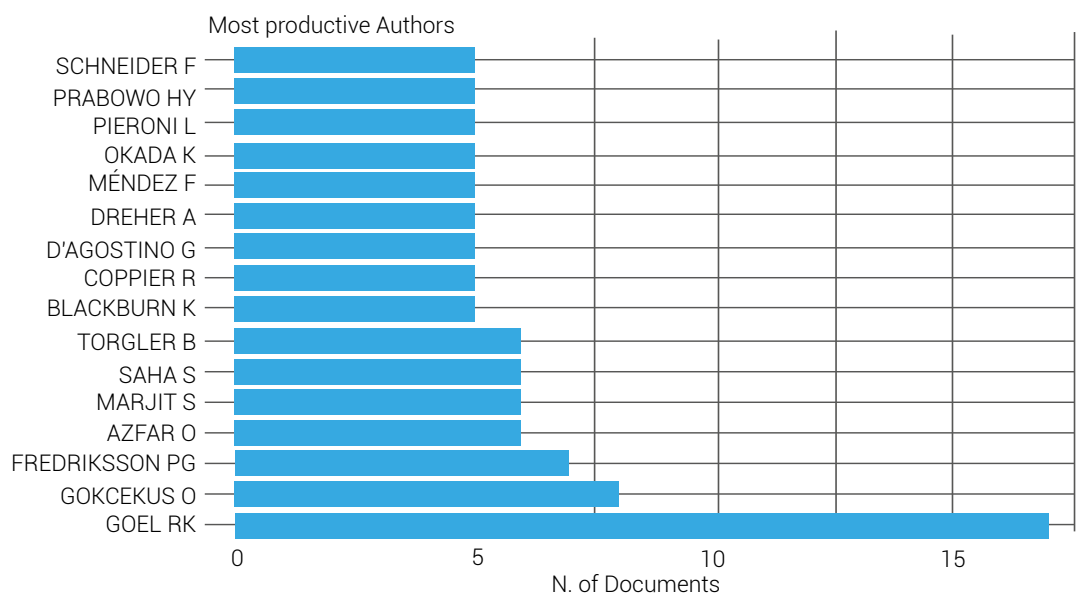

Figura 5. Principales investigadores.

Fuente: elaboración propia con base en información de SCOPUS, a través de biblioshiny. 
Con respecto a los principales investigadores, en la siguiente tabla se presenta su área de experiencia, vínculo organizacional e información de contacto:

Tabla 4. Ficha de caracterización de los principales investigadores.

\begin{tabular}{|l|l|l|l|l|}
\hline \multicolumn{1}{|c|}{ Investigador } & $\begin{array}{l}\text { Área de Investigación } \\
\text { Principal }\end{array}$ & Experiencia & \multicolumn{1}{|c|}{ Vinculación } & E-mail \\
\hline Goel RK & $\begin{array}{l}\text { Soborno, corrupción } \\
\text { política }\end{array}$ & $2009-2017$ & $\begin{array}{l}\text { Department of Economics, } \\
\text { Illinois State University }\end{array}$ & rkgoel@ilstu.edu \\
\hline $\begin{array}{l}\text { Fredriksson, } \\
\text { Per G. }\end{array}$ & $\begin{array}{l}\text { Instituciones, } \\
\text { desarrollo económico } \\
\text { yética }\end{array}$ & $2003-2018$ & $\begin{array}{l}\text { University of Louisville, } \\
\text { Department of Economics }\end{array}$ & per.fredriksson@louisville.edu \\
\hline $\begin{array}{l}\text { Gökçekuş, } \\
\text { Ömer }\end{array}$ & $\begin{array}{l}\text { Soborno, corrupción } \\
\text { política }\end{array}$ & $2006-2017$ & $\begin{array}{l}\text { Seton Hall University, } \\
\text { School of Diplomacy and } \\
\text { International Relations, }\end{array}$ & omer.gokcekus@shu.edu \\
\hline Méndez, Fabio & $\begin{array}{l}\text { Soborno, corrupción } \\
\text { política }\end{array}$ & $2006-2018$ & $\begin{array}{l}\text { Loyola University Maryland, } \\
\text { Sellinger School of Business } \\
\text { and Management }\end{array}$ & fmendez1@loyola.edu \\
\hline $\begin{array}{l}\text { Prabowo, } \\
\text { Hendi Yogi }\end{array}$ & $\begin{array}{l}\text { Educación, aprendizaje } \\
\text { transformativo }\end{array}$ & $2016-2018$ & $\begin{array}{l}\text { Universitas Islam Indonesia, } \\
\text { Accounting Programme }\end{array}$ & hendi_prabowo@yahoo.com \\
\hline
\end{tabular}

Fuente: elaboración propia con base en información de SCOPUS.

De otro lado, con base en el siguiente esquema de redes de autores, se identifican claramente 5 grupos de autores que trabajan en temas comunes.

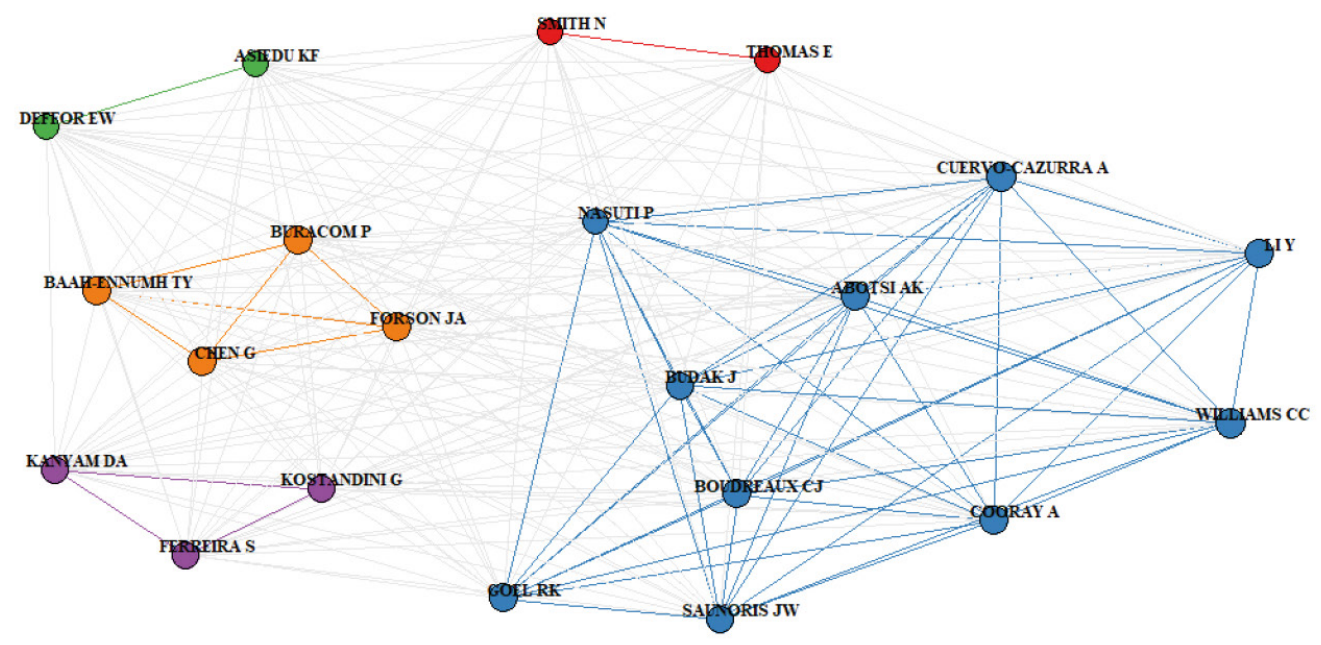

Figura 6. Redes de autores.

Fuente: elaboración propia con base en información de SCOPUS, a través de biblioshiny. 


\section{CONCLUSIONES}

Es importante destacar que las investigaciones científicas deben tener la importancia y compromiso continuo para cada uno de sus investigadores; contribuir y participar son factores que se complementan de manera continua para lograr ser parte de una sociedad informada y especializada teniendo en cuenta que la corrupción tiene sub-lineas (fiscalización, transparencia, control, entre otras); logrando así dar un ordenamiento al semillero de investigación y potencializando su capacidad de interacción con el medio actor. Finalmente, con base en la presente revisión científica se identificó que:

- Existe una tendencia creciente del interés de los investigadores sobre el tema.

- El liderazgo de la investigación lo ejercen instituciones como Ilinois State University y el World Bank.

- Las líneas de investigación para el semillero STATISTICS AND PROBABILITY deberían ser: anticorrupción, corrupción y crecimiento económico; corrupción y desarrollo; educación, ética y normas sociales; libertad económica y democracia.

- Los países que han presentado un mayor interés por estudiar el tema de la corrupción en el periodo de tiempo estudiado entre (1989-2018), son Estados Unidos, Reino Unido, Alemania, Italia y Francia.

- Las principales entidades financiadoras de las investigaciones científicas son: World Bank Group (WBG), National Natural Science Foundation of China (NSFC) y Economic and Social Research Council (ESRC).

\section{REFERENCIAS}

AENOR. (2018). UNE 166006:2018. Madrid: Asociación Española de Normalización.

Bada Carbajala, L. M., Rivas Tovar, L. A., y Littlewood Zimmermanc, H. F. (2017). Modelo de asociatividad en la cadena productiva en las Mipymes agroindustriales Model of associativity in the production chain in Agroindustrial SMEs. Contaduría y Administración, 1100-1117. 
Cely, N. C. (2004). Las Líneas de Investigación y la Formación de Investigadores: Una Mirada desde la Administración y sus Procesos Formativos. Bogotá.

Escorsa Castells, P. (2007). ¿Qué es la Inteligencia Competitiva? Madrid: Universidad Carlos III.

Garrido, M. F. (2003). Formación basada en las Tecnologías de la Información y Comunicación: Análisis Didáctico del Proceso de Enseñanza - Aprendizaje. Tarragona - España. Recuperado de https:// www.tdx.cat/bitstream/handle/10803/8909/Etesis_1.pdf

Grancolombiano, P. (s.f.). Protocolo para aprobación de líneas de investigación de las Facultades. Bogotá. doi:I+D+i-0008-5PCH014553

Isaza Castro, J. G. (2006). Cadenas productivas. Enfoques y precisiones conceptuales. Bogotá: Universidad Externado de Colombia.

Palop, F., y Vicente, J. M. (1999). Vigilancia tecnológica e inteligencia competitiva. Su potencial para la empresa española. Madrid: IMPIVA - COTEC.

Simanca, M. M., Montoya, L. A., y Bernal, C. A. (2016). Gestión del Conocimiento en Cadenas Productivas. El Caso de la Cadena Láctea en Colombia. Información tecnológica, 93-106.

Tiempo, E. (26 de 02 de 2017). El Tiempo. Obtenido de https://www.eltiempo.com/justicia/delitos/precio-de-la-corrupcion-en-colombia-61749 